\title{
Structural transformation in magnetite below the Verwey transition
}

\author{
Javier Blasco, Joaquín García, and Gloria Subías \\ Instituto de Ciencia de Materiales de Aragón, Departamento de Física de la Materia Condensada, CSIC_Universidad de Zaragoza, \\ E-50009 Zaragoza, Spain
}

(Received 25 January 2011; revised manuscript received 7 February 2011; published 18 March 2011)

\begin{abstract}
The magnetite structure was studied with synchrotron x-ray powder diffraction above and below the Verwey transition. A symmetry-mode analysis was performed to obtain the atomic displacements from the amplitudes of condensing modes. The main contributing modes that drive the structural phase transition at the Verwey temperature correspond to the irreducible representations $\Delta_{5}, X_{1}, X_{4}, W_{1}$, and $W_{2}$. The $W$ modes, neglected so far, must be taken into account so a reliable description of the low-temperature crystal structure can be obtained. This is refined in the nonpolar space group $C 2 / c$ with ten nonequivalent octahedral irons. The condensation of the mentioned modes leads to a wide distribution of local environments around the octahedral iron atoms, whose valences range between 2.53 and 2.84. This finding rules out any bimodal charge disproportionation of the octahedral iron atoms, i.e., an $\mathrm{Fe}^{2+}$-like/ $\mathrm{Fe}^{3+}$-like ordering.
\end{abstract}

DOI: 10.1103/PhysRevB.83.104105

PACS number(s): 71.30.+h, 61.50.Ks, 64.60.Bd, 71.45.Lr

\section{INTRODUCTION}

Magnetite is a ferrimagnetic mineral belonging to the family of spinels with inverse structure. The chemical formula for spinels is $A B_{2} \mathrm{O}_{4}$, and their crystal structures are cubic, space group $\mathrm{Fd} \overline{3} m$. The formula of magnetite is sometimes written as $\mathrm{Fe}^{3+}\left[\mathrm{Fe}^{3+} \mathrm{Fe}^{2+}\right] \mathrm{O}_{4}$ to reflect the fact that tetrahedral sites are occupied by trivalent ions, while the octahedral sites are filled by an equal mix of $\mathrm{Fe}^{3+}$ and $\mathrm{Fe}^{2+}$ ions. Magnetite is an electrical conductor at room temperature. Upon cooling, Verwey discovered a metal-insulator transition at $T_{\mathrm{V}} \sim 123 \mathrm{~K}$ characterized by a sudden decrease of two orders of magnitude in its electrical conductivity. ${ }^{1}$ Verwey ascribed this transition to electron localization on distinguishable octahedral sites below $T_{\mathrm{V}}$, minimizing the Coulomb repulsion among them, ${ }^{2}$ a feature known as the Anderson condition. ${ }^{3}$ The electron in question was assumed to fluctuate between the two octahedral sites for $T>T_{\mathrm{V}}$. Since then, this type of charge ordering (CO) has become the archetype for other metal-insulator transitions in mixed-valence oxides.

The picture of the Verwey transition, with a bimodal distribution of $\mathrm{Fe}^{2+}$ and $\mathrm{Fe}^{3+}$ ions on the octahedral sites, was soon in conflict with the properties of magnetite below $T_{\mathrm{V}}$ (see, for instance, Ref. 4). Neutron, x-ray, and electron diffraction studies on single crystals ${ }^{5-7}$ showed that the low-temperature cell is monoclinic with lattice vectors $(1,1,0),(-1,1,0)$, and $(0,0,2)$ with respect to the parent cubic structure. The most complete study, performed by Iizumi et al.,$^{5}$ proposed the space group $C c$ with 16 nonequivalent octahedral sites for the low-temperature cell. Formally, 16 different sites would correspond to 16 different charges. This result seemed to be confirmed by recent spectroscopic measurements, in which a ${ }^{57} \mathrm{Fe}$ NMR survey resolved 15 of the 16 octahedral environments. ${ }^{8}$ The differences among these octahedral sites must be small in accordance with previous resonant $\mathrm{x}$-ray scattering (RXS) experiments probing $(00 l)$ and $\left(00 \frac{l}{2}\right)$ reflections. ${ }^{9,10}$ The Verwey transition was recently studied by inelastic neutron scattering, and a large splitting in the acoustic wave branch at $T_{\mathrm{V}}$ with the appearance of a gap at $\mathbf{q}=(0,0,1 / 2)$ was observed. No CO model was able to account for the experimental splitting, and the formation of a charge-density wave was suggested. ${ }^{11}$

However, a thorough work by Wright et al. ${ }^{12,13}$ suggests a different $\mathrm{CO}$ scheme. Using $\mathrm{x}$-ray and neutron powder diffraction, they refined the structure in the space group $P 2 / c$ with the following lattice vectors with respect to the cubic structure: $(1 / 2,1 / 2,0),(-1 / 2,1 / 2,0)$, and $(0,0,2)$. The primitive unit cell for the phase $P 2 / c$ contains six nonequivalent octahedral sites, denoted as $B 1 a, B 1 b, B 2 a, B 2 b, B 3$, and $B 4$. Through the orthorhombic Pmca pseudosymmetry constraint, $B 1 a$ and $B 1 b$ became symmetry equivalent; they are labeled $B 1$ in Ref. 12. The same argument applies to the $B 2 a$ and $B 2 b$ sites. Relationships between the above-mentioned unit cells $(\mathrm{Fd} \overline{3} m, P 2 / c$ or $P m c a$, and $C c$ or $C 2 / c)$ are illustrated in Fig. 1. Over the four octahedral sites (denoted as $B 1, B 2, B 3$, and B4), Wright et al.. ${ }^{12,13}$ obtained an arrangement of $\mathrm{Fe}^{+2.4}$ and $\mathrm{Fe}^{+2.6}$ that does not satisfy the Anderson condition but which has a charge modulation along the $z$ direction. Despite the fact that this structure is only a first approach, accounting for only a quarter of the actual cell, recent calculations still use it to explain the Verwey transition within a scheme of pseudo- $\mathrm{Fe}^{2+} / \mathrm{Fe}^{3+}$ charge and orbital orderings. ${ }^{14-18}$

To sum up, the lack of an accurate determination of the magnetite structure below $T_{\mathrm{V}}$ has obviated a reliable characterization of the $\mathrm{CO}$ developed at the metal-insulator transition, if any. This is because of the enormous difficulty involved in solving its crystal structure at low temperature. For one thing, the proposed monoclinic cell contains 32 formula units, which implies a large number of parameters to be accounted for by conventional powder diffraction. Moreover, the phase transition leads to strong twinning, which, in addition to the presence of multiple scattering and self-absorption problems, makes structure resolution by single-crystal diffraction very difficult. The most complete structural studies have overcome these difficulties by making use of constraints in the refinement processes. Iizumi et al..$^{5}$ and Wright et al. ${ }^{12}$ realized that the most intense superstructure peaks at low temperature come from the condensation of $\Delta_{5}$ and $X_{1}$ modes, which allows stable refinements using structural constraints arising from the transition of the cubic Fd $\overline{3} m$ to the orthorhombic Pmca cell. 


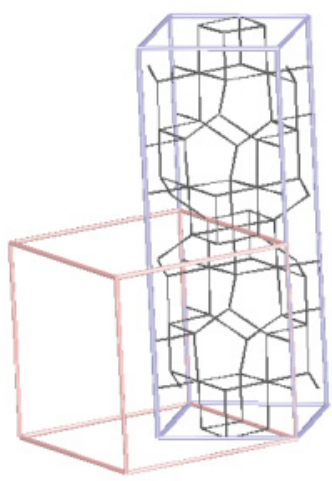

Pmca $-P 2 / c$

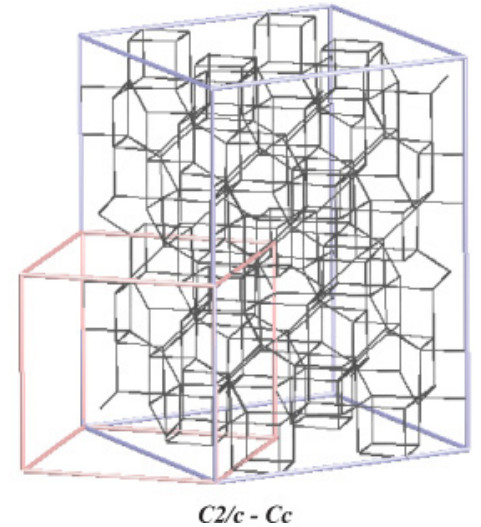

$C 2 / c-C c$
FIG. 1. (Color online) Relationship between the parent cubic structure of a spinel and the distorted structures referred to in the text. Left-hand side: Distorted structure (Pmca or $P 2 / c$ ) with lattice vectors $\left(\frac{1}{2}, \frac{1}{2}, 0\right),\left(-\frac{1}{2}, \frac{1}{2}, 0\right)$, and $(0,0,2)$ with respect to the cubic structure. Right-hand side: Distorted structures $(C 2 / c$ or $C / c)$ with lattice vectors $(1,1,0),(-1,1,0)$ and $(0,0,2)$. The bonds are also shown in the distorted structures. The figures were obtained from ISODISPLACE (Ref. 20).

This approach has the disadvantage of masking any type of charge modulation within the monoclinic $a b$ plane.

In order to obtain an accurate picture of the crystal structure below $T_{\mathrm{V}}$, we have carried out a structural study using high-resolution synchrotron x-ray powder diffraction data from a highly stoichiometric powder of magnetite. The paper is organized as follows: Sec. II explains the physical basis of the structure analysis; experimental details are collected in Sec. III; results and discussion are reported in Sec. IV; and Sec. V summarizes the main conclusions of this work.

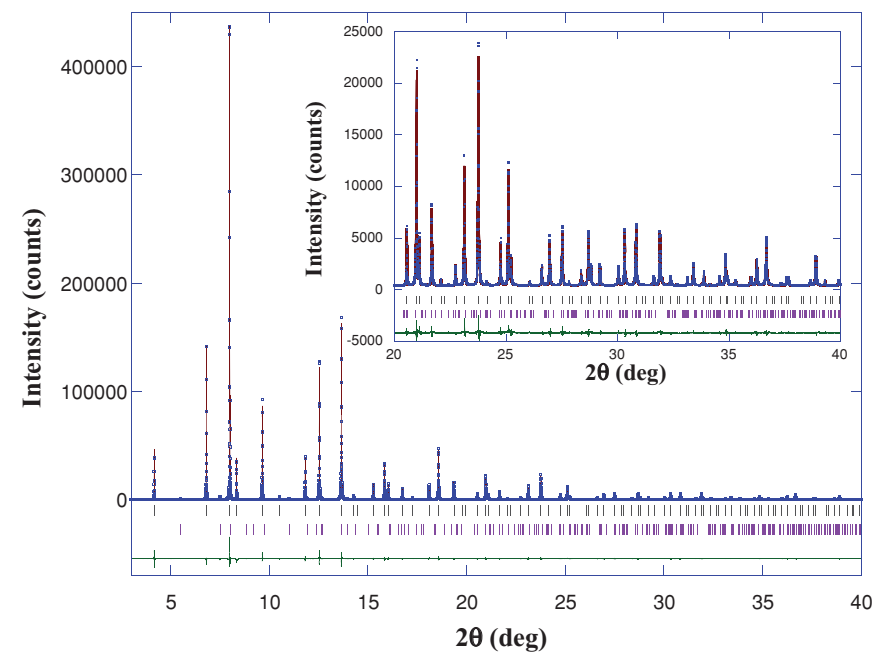

FIG. 2. (Color online) Observed (points), calculated (line), and difference (bottom line) of the Rietveld refinement of magnetite at $160 \mathrm{~K}$. The bars indicate the allowed Bragg reflections and the second row corresponds to the impurity of hematite. Inset: Detail of the same refinement at high angles.

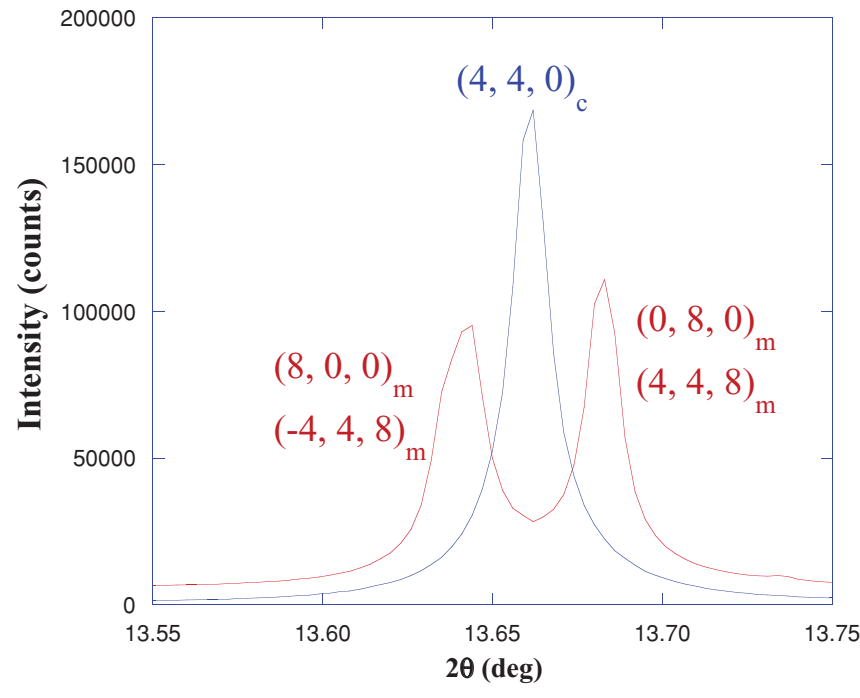

FIG. 3. (Color online) An example of the peak splitting observed in the comparison of the patterns taken at 160 and $80 \mathrm{~K}$. The latter has been shifted upward for clarity. The subscripts $\mathrm{c}$ and $\mathrm{m}$ on the Miller indices identify cubic and monoclinic cells, respectively.

\section{MODE ANALYSIS}

In a structural phase transition, the low-temperature phase (or distorted structure) can be described as the hightemperature phase (or parent structure) modified by static frozen distortions coming from atomic displacements. In the Landau theory, the main signature of a phase transition is the appearance of a symmetry-breaking distortion, which is mainly caused by the freezing of symmetry-adapted modes associated with the order parameter. ${ }^{19}$ The frozen distortions are accounted for by modes that are collective correlated atomic displacements fulfilling specific symmetry properties. Any structural distortion can be decomposed into contributions of modes with different symmetries given by irreducible

TABLE I. Refinement parameters: temperature, space group, unit-cell parameters, and reliability factors. The number of variables and reflections corresponds to the combination of the two phases, and $d$-spacing range indicates the location of the last and first reflections in the refined range. Numbers in parentheses are standard deviations in the least significant digit.

\begin{tabular}{lcc}
\hline \hline$T(\mathrm{~K})$ & 160 & 80 \\
\hline Space group & $\mathrm{Fd} \overline{3} m$ & $C 2 / c$ \\
\hline$a(\AA)$ & $8.39457(1)$ & $11.89310(1)$ \\
$b(\AA)$ & - & $11.85418(2)$ \\
$c(\AA)$ & - & $16.78310(2)$ \\
$\beta($ deg $)$ & - & $90.2367(2)$ \\
No. of variables & 15 & 67 \\
No. of reflections & 727 & 10649 \\
$d$-spacing range $(\AA)$ & $0.4176-4.8466$ & $0.4924-5.9465$ \\
$R_{\mathrm{wp}}$ & 7.2 & 7.9 \\
$R_{\mathrm{p}}$ & 5.6 & 6.0 \\
$R_{\text {Bragg }}$ & 2.6 & 3.5 \\
$\chi^{2}$ & 5.3 & 4.0 \\
\hline \hline
\end{tabular}


representations (irrep) of the parent space group. A space group irrep can only induce distortions that produce the reciprocal superlattice defined by its $k$ point. Therefore, the reciprocal-space $k$ point or wave vector of a phase transition indicates the location of any superlattice diffraction peaks that may arise in the distorted phase.

When a crystal experiences a symmetry-lowering structural distortion, some of the symmetry elements of the parent space group are lost, while the rest persist in the resulting superstructure. The persisting elements form the space-group symmetry of the low-symmetry phase, which is called an isotropy subgroup of the parent structure. The distortion relating parent structures to distorted ones can be divided into two parts: first, a strain of the parent lattice, and second, a set of atomic displacements, which may break some translational symmetry without affecting the metrics of the underlying parent lattice. The first part is accounted for by refining the actual cell obtained from the diffraction experiments. The atomic position of an $i$ atom in the distorted structure for a given irrep can be expressed as $\vec{r}_{i}=\vec{r}_{i}^{0}+\vec{u}_{i}$, where $\vec{r}_{i}^{0}$ is the $i$-atom position of the parent structure expressed in the setting of the space group for the distorted structure. $\vec{u}_{i}$ is the atomic displacement, which can be expressed as a linear combination of a basis of symmetry-adapted modes, $\vec{u}_{i}=$ $\sum_{j=1}^{n} Q_{j} \vec{\varepsilon}_{j}$, where $Q_{j}$ stands for an amplitude with units of length and $\vec{\varepsilon}_{j}$ are the components of the so-called polarization vector, which describes the corresponding set of correlated atomic displacements. This set of normalized displacements $\vec{\varepsilon}\left(\vec{\varepsilon}_{1}, \vec{\varepsilon}_{2}, \vec{\varepsilon}_{3}, \ldots, \vec{\varepsilon}_{n}\right)$ within the asymmetric unit of the isotropy subgroup unambiguously defines the polarization vector of the corresponding symmetry mode. The displacements for the rest of the atoms are obtained by the symmetry operations of the low-temperature space group. Once the decomposition of the proposed structure in terms of symmetry modes is done, the free parameters become $Q_{j}$ instead of individual atomic coordinates, and this procedure allows more stable

TABLE II. Wyckoff positions, refined fractional coordinates, isotropic temperature factors and bond valence sums. An average temperature factor was refined for all atoms at $80 \mathrm{~K}$. Letters and numbers are indicated in the atom column for comparison with Ref. 13. Numbers in parentheses for the rest of the columns are standard deviations in the least significant digit.

\begin{tabular}{|c|c|c|c|c|c|c|}
\hline Atom & Site & $x / a$ & $y / b$ & $z / c$ & $B\left(\AA^{2}\right)$ & BVS \\
\hline \multicolumn{7}{|c|}{ Cubic cell at $160 \mathrm{~K}$} \\
\hline $\mathrm{Fe}(A)$ & $8 b$ & $1 / 8$ & $1 / 8$ & $1 / 8$ & $0.321(3)$ & $2.861(2)$ \\
\hline $\mathrm{Fe}(B)$ & $16 c$ & $1 / 2$ & $1 / 2$ & $1 / 2$ & $0.439(2)$ & $2.649(1)$ \\
\hline $\mathrm{O}$ & $32 e$ & $0.25451(6)$ & $0.25451(6)$ & $0.25451(6)$ & $0.429(8)$ & $2.040(1)$ \\
\hline \multicolumn{7}{|c|}{ Monoclinic cell at $80 \mathrm{~K}$} \\
\hline $\mathrm{Fe}(A 1)$ & $8 f$ & $0.12413(14)$ & $0.0030(4)$ & $0.06416(17)$ & $0.258(2)$ & $2.86(3)$ \\
\hline $\mathrm{Fe}(A 1)$ & $8 f$ & $0.62587(14)$ & $0.0006(4)$ & $0.06242(17)$ & $0.258(2)$ & $2.86(3)$ \\
\hline $\mathrm{Fe}(A 2)$ & $8 f$ & $0.3747(3)$ & $0.7538(3)$ & $0.3134(3)$ & $0.258(2)$ & $2.79(4)$ \\
\hline $\mathrm{Fe}(A 2)$ & $8 f$ & $0.3753(3)$ & $0.2521(3)$ & $0.3100(3)$ & $0.258(2)$ & $2.83(4)$ \\
\hline $\operatorname{Fe}(B 1 a)$ & $8 f$ & $-0.0023(4)$ & $0.7474(2)$ & $0.5005(3)$ & $0.258(2)$ & $2.535(32)$ \\
\hline $\mathrm{Fe}(B 1 b)$ & $4 c$ & $3 / 4$ & $1 / 4$ & $1 / 2$ & $0.258(2)$ & $2.68(3)$ \\
\hline $\mathrm{Fe}\left(B 1 b^{\prime}\right)$ & $4 d$ & $3 / 4$ & $1 / 4$ & 0 & $0.258(2)$ & $2.53(3)$ \\
\hline $\mathrm{Fe}(B 2 a)$ & $4 e$ & $1 / 2$ & $0.0027(4)$ & $1 / 4$ & $0.258(2)$ & $2.83(3)$ \\
\hline $\mathrm{Fe}\left(B 2 a^{\prime}\right)$ & $4 e$ & 0 & $0.0068(4)$ & $1 / 4$ & $0.258(2)$ & $2.805(29)$ \\
\hline $\mathrm{Fe}(B 2 b)$ & $8 f$ & $0.25088(8)$ & $0.0048(2)$ & $0.25088(8)$ & $0.258(2)$ & $2.84(3)$ \\
\hline $\mathrm{Fe}(B 3)$ & $8 f$ & $0.3748(3)$ & $0.6309(2)$ & $0.1192(2)$ & $0.258(2)$ & $2.61(3)$ \\
\hline $\mathrm{Fe}\left(B 3^{\prime}\right)$ & $8 f$ & $0.1248(3)$ & $0.3657(2)$ & $0.8803(2)$ & $0.258(2)$ & $2.79(3)$ \\
\hline $\mathrm{Fe}(B 4)$ & $8 f$ & $0.6222(2)$ & $0.6240(3)$ & $0.8762(2)$ & $0.258(2)$ & $2.69(3)$ \\
\hline $\mathrm{Fe}\left(B 4^{\prime}\right)$ & $8 f$ & $0.1278(2)$ & $0.3776(2)$ & $0.3749(2)$ & $0.258(2)$ & $2.53(3)$ \\
\hline $\mathrm{O}(1)$ & $8 f$ & $0.3720(9)$ & $0.1269(7)$ & $0.5056(6)$ & $0.258(2)$ & $2.10(3)$ \\
\hline $\mathrm{O}(1)$ & $8 f$ & $0.1230(9)$ & $0.1320(7)$ & $0.9994(6)$ & $0.258(2)$ & $2.04(3)$ \\
\hline $\mathrm{O}(2)$ & $8 f$ & $0.6261(9)$ & $0.8722(7)$ & $0.9975(6)$ & $0.258(2)$ & $2.15(3)$ \\
\hline $\mathrm{O}(2)$ & $8 f$ & $0.3750(9)$ & $0.3712(7)$ & $0.5015(6)$ & $0.258(2)$ & $2.08(3)$ \\
\hline $\mathrm{O}(3)$ & $8 f$ & $0.6142(9)$ & $0.6311(10)$ & $0.2573(7)$ & $0.258(2)$ & $1.95(3)$ \\
\hline $\mathrm{O}(3)$ & $8 f$ & $0.1347(9)$ & $0.6187(10)$ & $0.2570(7)$ & $0.258(2)$ & $2.05(3)$ \\
\hline $\mathrm{O}(4)$ & $8 f$ & $0.3683(9)$ & $0.3905(10)$ & $0.2468(7)$ & $0.258(2)$ & $2.00(3)$ \\
\hline $\mathrm{O}(4)$ & $8 f$ & $0.3827(9)$ & $0.8841(10)$ & $0.2526(7)$ & $0.258(2)$ & $2.05(3)$ \\
\hline $\mathrm{O}(5 a)$ & $8 f$ & $0.4973(3)$ & $0.0075(13)$ & $0.1292(3)$ & $0.258(2)$ & $1.92(2)$ \\
\hline $\mathrm{O}(5 a)$ & $8 f$ & $0.0027(3)$ & $0.0031(13)$ & $0.8708(3)$ & $0.258(2)$ & $2.06(2)$ \\
\hline$O(5 b)$ & $8 f$ & $0.2501(4)$ & $0.0081(13)$ & $0.1319(4)$ & $0.258(2)$ & $2.00(2)$ \\
\hline $\mathrm{O}(5 b)$ & $8 f$ & $0.7561(4)$ & $-0.0037(13)$ & $0.1259(4)$ & $0.258(2)$ & $2.00(2)$ \\
\hline $\mathrm{O}(6 a)$ & $8 f$ & $0.4985(8)$ & $0.2497(7)$ & $0.3755(6)$ & $0.258(2)$ & $2.10(3)$ \\
\hline $\mathrm{O}(6 a)$ & $8 f$ & $0.9896(8)$ & $0.2557(7)$ & $0.1243(6)$ & $0.258(2)$ & $2.08(3)$ \\
\hline$O(6 b)$ & $8 f$ & $0.2420(8)$ & $0.7527(6)$ & $0.3781(6)$ & $0.258(2)$ & $2.12(3)$ \\
\hline $\mathrm{O}(6 b)$ & $8 f$ & $0.7502(8)$ & $0.2527(6)$ & $0.1262(6)$ & $0.258(2)$ & $2.04(3)$ \\
\hline
\end{tabular}


TABLE III. Irreducible representations with possible frozen modes for the indicated distorted structures of the parent cubic spinel.

\begin{tabular}{ll}
\hline \hline Distorted structure & \multicolumn{1}{c}{ irreps } \\
\hline Pmca & $\Gamma_{1}^{+} ; \Gamma_{3}^{+} ; \Gamma_{5}^{+} ; \Delta_{5} ; X_{1}$ \\
$P 2 / c$ & $\Gamma_{1}^{+} ; \Gamma_{3}^{+} ; \Gamma_{4}^{+} ; \Gamma_{5}^{+} ; \Delta_{2} ; \Delta_{4} ; \Delta_{5} ; X_{1} ; X_{3}$ \\
$C 2 / c$ & $\Gamma_{1}^{+} ; \Gamma_{3}^{+} ; \Gamma_{4}^{+} ; \Gamma_{5}^{+} ; \Delta_{2} ; \Delta_{4} ; \Delta_{5} ; X_{1} ; X_{2} ; X_{3} ; X_{4} ; W_{1} ; W_{2}$ \\
$C c$ & $\Gamma_{1}^{+} ; \Gamma_{3}^{+} ; \Gamma_{4}^{+} ; \Gamma_{5}^{+} ; \Gamma_{2}^{-} ; \Gamma_{4}^{-} ; \Gamma_{5}^{-} ; \Delta_{2} ; \Delta_{4} ; \Delta_{5} ; X_{1} ; X_{2} ; X_{3} ; X_{4} ; W_{1} ; W_{2}$ \\
\hline \hline
\end{tabular}

and robust refinements. The normalization of the eigenvectors for each irrep allows the comparison of their amplitudes and hence indicates their importance in the mechanism of the Verwey transition. Therefore, this method is especially useful for treating the magnetite structure below $T_{\mathrm{V}}$, in which a great number of atomic positions are free parameters; their displacements are correlated through the mode analysis.

Nowadays there are programs such as ISODISPLACE ${ }^{20}$ and AMPLIMODES $^{21}$ able to yield individual mode amplitudes that can be passed to Rietveld refinement programs. Finally, the refined amplitudes can be expressed in conventional fractional coordinates. Further details about the theory of the symmetry-mode analysis and the available tools can be found elsewhere. $^{22,23}$

\section{EXPERIMENTAL SECTION}

Magnetite was prepared with high-purity $\mathrm{Fe}_{2} \mathrm{O}_{3}(99.999 \%)$. The oxide was fired at $1200{ }^{\circ} \mathrm{C}$ for $24 \mathrm{~h}$ in a current flow of $\mathrm{CO}_{2} / \mathrm{CO}(96 / 4)$. The powders were ground, pressed into rods, and sintered at $1400{ }^{\circ} \mathrm{C}$ for $24 \mathrm{~h}$ in the same atmosphere, ensuring the right cation-to-oxygen stoichiometry. ${ }^{24}$ Single crystals of magnetite were grown from the rods in the same atmosphere by the floating zone method. ${ }^{25}$ The final powder of magnetite was obtained by grinding the single crystals. Single crystals showed a saturation magnetization at $5 \mathrm{~K}$ of $4.11 \mu_{\mathrm{B}}$, and $T_{\mathrm{V}}$ is $123.5 \mathrm{~K}$ with a narrow transition width $(\Delta T=1 \mathrm{~K}){ }^{26}$
These features correspond to highly stoichiometric samples, $\mathrm{Fe}_{3(1-\delta)} \mathrm{O}_{4}$ with $\delta \cong 0.000 .^{24,27,28}$

Synchrotron $\mathrm{X}$-ray diffraction patterns were measured at the ID31 beamline at the ESRF (Grenoble, France). This is an insertion device with small-gap undulators and fine tuning of the magnets to permit both high-brilliance $\mathrm{x}$ rays and high collimation in the horizontal and vertical directions. The result is a significant increase in the flux incident on the sample and an improvement in the signal-to-noise ratio. ${ }^{29}$ The sample was loaded in a borosilicate glass capillary $(\phi=0.5 \mathrm{~mm})$ and kept spinning during data acquisition. A short wavelength, $\lambda=0.3528 \AA$, was selected to reduce absorption. The value of $\lambda$ was calibrated using standard silicon. The data were collected at 80 and $160 \mathrm{~K}$ for $6 \mathrm{~h}$ at each temperature.

Diffraction patterns were analyzed by the Rietveld method using the FULLPROF program ${ }^{30}$ with the symmetry-mode analysis. The input file with the basis modes was obtained from the AMPLIMODES program. ${ }^{21,23}$

\section{RESULTS AND DISCUSSION}

Our sample adopts the expected cubic structure with space group $\mathrm{Fd} \overline{3} m$ at $160 \mathrm{~K}$. The diffraction pattern also reveals the presence of traces of hematite, $0.5(1) \mathrm{wt} \%$. This weak contribution from the hematite phase was also detected in previous works, and it was ascribed to the surface oxidation of the powder during the grinding process. ${ }^{12}$ The impurity was

TABLE IV. Mode decomposition with respect to $\mathrm{Fd} \overline{3} m$ of the $C 2 / c$ structure of magnetite. The respective $k$ points, irrep, and isotropy subgroup are summarized, together with the dimension (i.e., number of individual modes for each irrep). The global distortion amplitudes correspond to the fractional coordinates indicated in Ref. $13\left(A_{1}\right)$, the refinement of all allowed modes with Pmca constraints $\left(A_{2}\right)$, and the last refinement without $P m c a$ constraints $\left(A_{3}\right)$.

\begin{tabular}{|c|c|c|c|c|c|c|}
\hline$k$ point & irrep & Isotropy subgroup & Dimension & $A_{1}(\AA)$ & $A_{2}(\AA)$ & $A_{3}(\AA)$ \\
\hline$(0,0,0)$ & $\Gamma_{1}^{+}$ & $\mathrm{Fd} \overline{3} m(227)$ & 1 & 0.04 & 0.03 & 0.03 \\
\hline$(0,0,0)$ & $\Gamma_{3}^{+}$ & I4 $1 /$ amd (141) & 1 & 0.03 & 0.07 & 0.07 \\
\hline$(0,0,0)$ & $\Gamma_{4}^{+}$ & $C 2 / m(12)$ & 1 & 0.00 & 0.00 & 0.00 \\
\hline$(0,0,0)$ & $\Gamma_{5}^{+}$ & $C 2 / m(12)$ & 6 & 0.12 & 0.09 & 0.09 \\
\hline$(0,1 / 2,0)$ & $\Delta_{2}$ & Pcca (54) & 3 & 0.00 & 0.00 & 0.00 \\
\hline$(0,1 / 2,0)$ & $\Delta_{4}$ & $\operatorname{Pcca}(54)$ & 3 & 0.00 & 0.00 & 0.00 \\
\hline$(0,1 / 2,0)$ & $\Delta_{5}$ & Pbcm (57) & 11 & 0.48 & 0.48 & 0.53 \\
\hline$(0,1,0)$ & $X_{1}$ & $C 2 / m(12)$ & 14 & 0.33 & 0.34 & 0.31 \\
\hline$(0,1,0)$ & $X_{2}$ & $C 2 / m(12)$ & 3 & 0.00 & 0.00 & 0.00 \\
\hline$(0,1,0)$ & $X_{3}$ & $C 2 / m(12)$ & 10 & 0.00 & 0.00 & 0.00 \\
\hline$(0,1,0)$ & $X_{4}$ & $C 2 / m(12)$ & 6 & 0.00 & 0.00 & 0.31 \\
\hline$(1 / 2,1,0)$ & $W_{1}$ & $C 2 / m(12)$ & 10 & 0.00 & 0.00 & 0.40 \\
\hline$(1 / 2,1,0)$ & $W_{2}$ & $C 2 / m(12)$ & 11 & 0.00 & 0.00 & 0.49 \\
\hline
\end{tabular}


included in the structure refinements as a secondary phase. The calculated and experimental patterns are shown in Fig. 2, and details of the Rietveld refinement are given in Table I. Atomic coordinates and valence states obtained from bond valence sums ${ }^{31}$ (BVSs) are collected in Table II. The values obtained for the cubic phase agree with the reported data, ${ }^{13}$ and BVS indicates that tetrahedral irons are underbonded, i.e., the calculated valence is lower than the ideal one, whereas the octahedral atoms are overbonded.

Below the Verwey transition, two changes occur in the x-ray diffraction pattern. One is peak splitting in many of the strong diffraction lines (see Fig. 3), and the second is the appearance of new superstructure peaks with very weak intensity. All of these features can be accounted for by a monoclinic cell with the lattice parameters $\vec{a}_{\mathrm{m}}=\vec{a}_{\mathrm{c}}+\vec{b}_{\mathrm{c}}, \vec{b}_{\mathrm{m}}=\vec{b}_{\mathrm{c}}-\vec{a}_{\mathrm{c}}$, and $\vec{c}_{\mathrm{m}}=2 \vec{c}_{\mathrm{c}}$ (subscripts $\mathrm{m}$ and $\mathrm{c}$ refer to monoclinic and cubic cells, respectively). The reflection conditions concur with a $C$-centered lattice and the existence of a $c$-glide plane of symmetry. We have used the ISODISPLACE tool ${ }^{20}$ to explore the possible distortions capable of yielding the mentioned monoclinic cell. Two possible distorted structures are found with the polar $C c$ and the nonpolar $C 2 / c$ space groups. The cells are metrically equivalent for the two cases. ISODISPLACE and AMPLIMODES were used to decompose the symmetry-breaking distortion present in the two transitions-from $\mathrm{Fd} \overline{3} m$ to $C c$ and from $\mathrm{Fd} \overline{3} m$ to $C 2 / c$. A summary of the mode decomposition is collected in Table III. Both transitions have in common the active modes corresponding to the irreps $\Gamma_{1,3,4,5}^{+}, \Delta_{2,4,5}$, $X_{1,2,3,4}$, and $W_{1,2}$ (hereafter, we denote these modes as $\Gamma$ modes, $\Delta$ modes, and so on). The only difference concerns the modes belonging to irreps that break the inversion center, $\Gamma_{2,4.5}^{-}$, which are active in the transition to $C c$ but absent in the transition to $C 2 / c$. Accordingly, the $C 2 / c$ cell is a good nonpolar approximation for the $C c$ cell. Moreover, the $\mathrm{Fd} \overline{3} m$ -to- $C c$ transition has a total of 168 mode amplitudes to be refined, while the amplitudes involved in the transition to $C 2 / c$ are only 80 in number. On the basis of these considerations, we used $C 2 / c$ for the refinement of the $80-\mathrm{K}$ diffraction pattern.

The very weak superstructure peaks and the large number of structural variables led to unconstrained refinements that were not stable, diverging rapidly. Therefore, a stepwise refinement strategy was adopted and the number of degrees of freedom was gradually increased. Previous studies pointed out the robustness of refinements using the symmetry constraints of the Pmca cell (standard setting Pbcm)..$^{5}$ This approach was also used in the refinement with a monoclinic $P 2 / c$ cell. ${ }^{12}$ The mode decompositions of these two distortions are also collected in Table III. We started our study by reproducing the refinement of the $P 2 / c$ model. ${ }^{12,13}$ Table IV shows the mode decomposition for the reported $P 2 / c$ structure in the setting of the $C 2 / c$ space group ( $A_{1}$ amplitudes). Here, a clear hierarchy can be established: $\Delta_{5}$ and $X_{1}$ are the primary modes; $\Gamma$ modes are secondary ones, accompanying the main transition. Subsequently, we completed the refinements of all the common active modes that are also present in the transition from the cubic $\operatorname{Fd} \overline{3} m$ to the Pmca cell. This refinement yielded the global distortion amplitudes collected in the sixth column of Table IV $\left(A_{2}\right)$. The small differences between $A_{1}$ and $A_{2}$ come from the refinement of additional modes
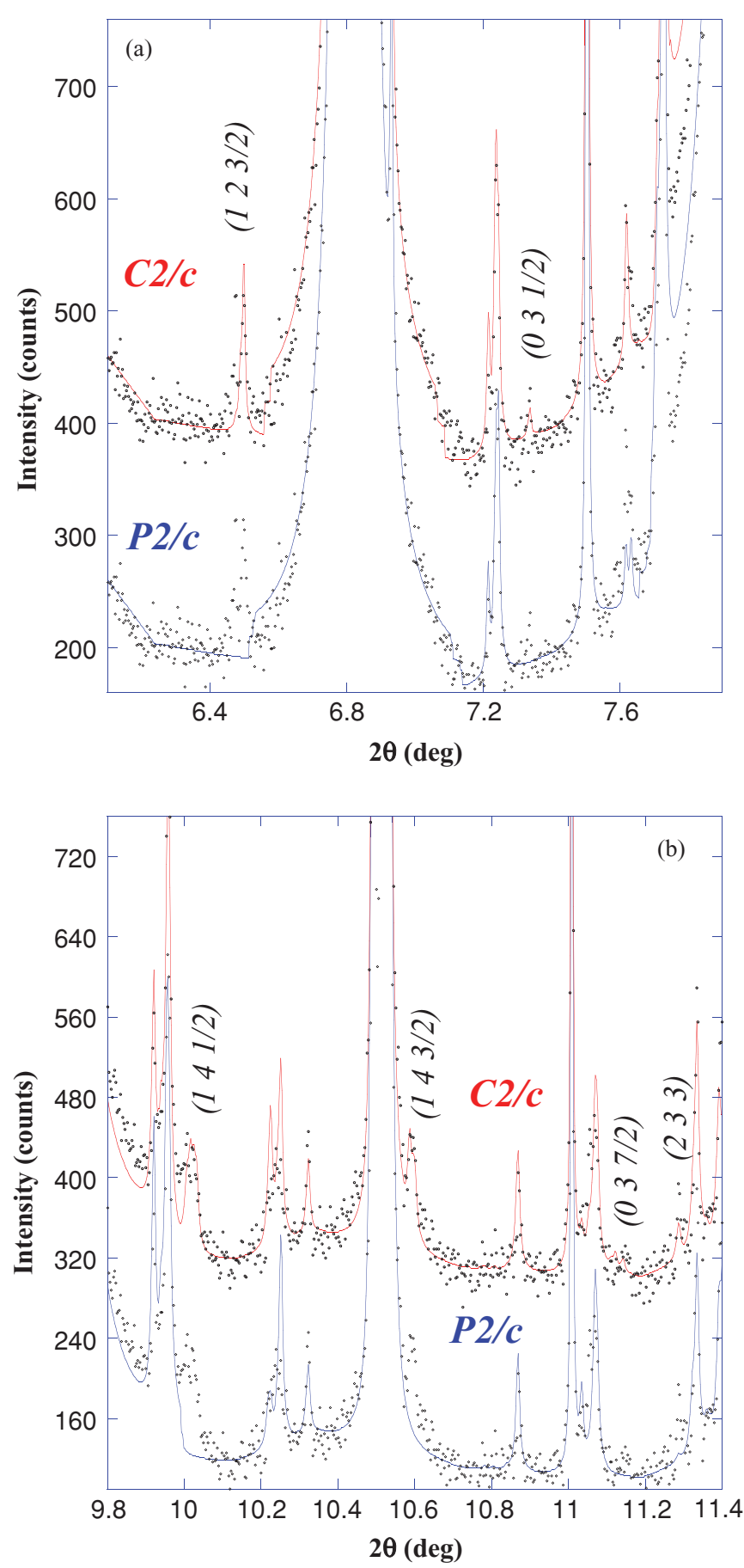

FIG. 4. (Color online) Comparison of the Rietveld refinements of magnetite at $80 \mathrm{~K}$ using the $P 2 / c$ and $C 2 / c$ models. The indicated Miller indices are some of the reflections not allowed by the $P 2 / C$ cell. The refinement with the $P 2 / c$ model is shifted downward for the sake of comparison.

(mainly involving to tetrahedral iron atoms) not considered in the constraints of Ref. 13. Both refinements yielded similar fractional coordinates and, therefore, similar bond lengths. However, they account for only approximately three-fourths of the weak superstructure peaks, with the weakest peaks still not fitted, as seen in Fig. 4. We then fixed the values of these amplitudes and refined additional $W$ modes associated with 


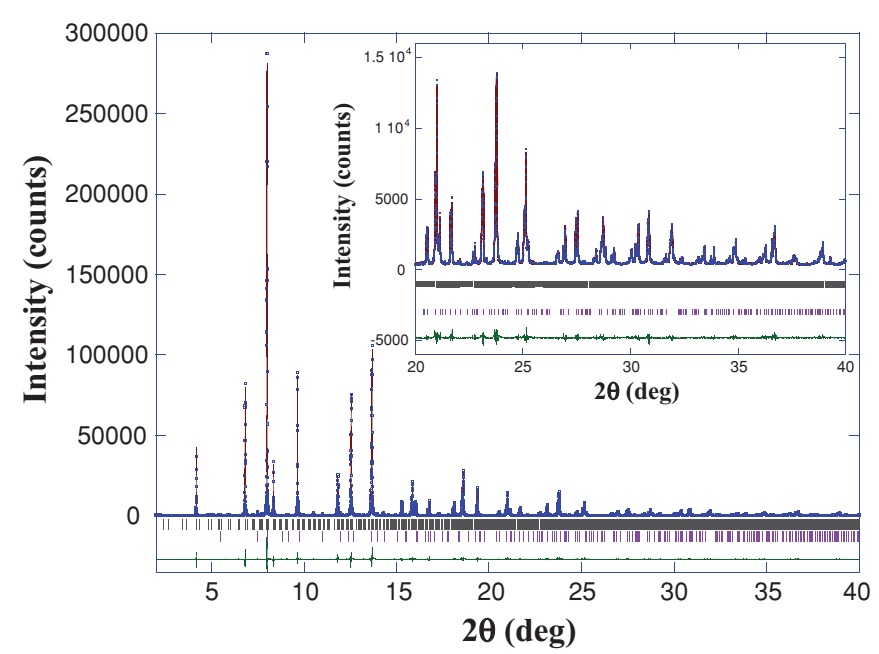

FIG. 5. (Color online) Observed (points), calculated (line), and difference (bottom line) of the Rietveld refinement of magnetite at 80 $\mathrm{K}$. The bars indicate the allowed Bragg reflections, and the second row corresponds to the impurity of hematite. Inset: Detail of the same refinement at high angles.

the $\left(\frac{1}{2}, 1,0\right) \boldsymbol{k}$ vector; i.e., we removed the Pmca constraints. Refining both $W_{1}$ and $W_{2}$, we were successful in explaining all superstructure peaks (see Fig. 4). The next step was to test if further modes had a role, but with the previously refined modes fixed. We found that only some modes of the irrep $X_{4}$ slightly improved the refinement, while the contribution from the remaining irreps $\left(\Gamma_{4}^{+}, \Delta_{2,4}\right.$, and $\left.X_{2,3}\right)$ was negligible. Finally, all amplitudes of contributing modes were refined freely, together with the remaining parameters (lattice, average temperature factor, and peak profile parameters) to verify the stability of the refinement. The final result of the refinement is plotted in Fig. 5. Details of the refinement and the resulting fractional coordinates are summarized in Tables I and II.

The fractional coordinates in Table II correspond to the global amplitudes given in the seventh column of Table IV $\left(A_{3}\right) . \Delta_{5}$ continues to be the most intense mode, but we observe that the refined amplitudes of $W$ and $X$ modes are equivalent, so there is no justification for neglecting the contribution of the former. It is worth comparing our results with the previous analysis in the $P 2 / c$ cell with half values for the $a$ - and $b$-monoclinic cell parameters. In $P 2 / c$, there are six nonequivalent octahedral sites but, due to the Pmca constraints, Wright et $a l{ }^{12}$ resolved only four sites: $B 1$ ( $a$ and $\left.b\right), B 2(a$ and $b), B 3$, and $B 4$. In the larger $C 2 / c$ cell, $B 1 b, B 2 a, B 3$, and $B 4$ are split into two nonequivalent sites, giving rise to a total of ten nonequivalent sites for octahedral irons. These yield ten different environments for these atoms, resulting in ten different valences (see Table II). The valences are very similar for some sites, as occurs for B2. Other sites are composed of an even mix of valences, as occurs for $B 1 b, B 3$, and $B 4$. Our results agree with the reported charge modulation between $B 1$ and $B 2$ sites along the $z$ direction. ${ }^{12,13}$ However, the valences of the $B 3$ and $B 4$ sites are strongly overlapped, and the average difference between the two sites is significantly smaller than that obtained from the $P 2 / c$ model. The spread of valences
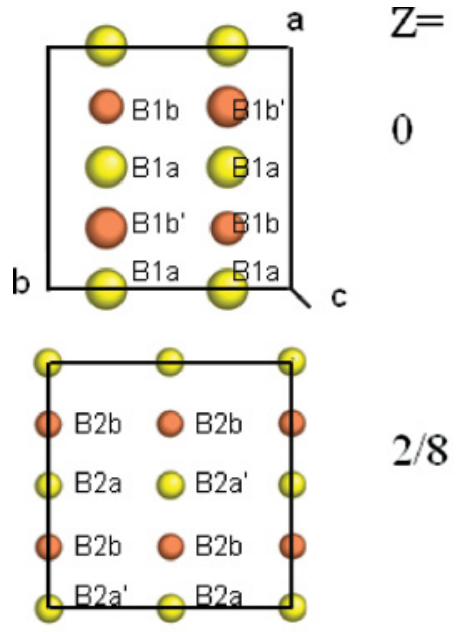

$2 / 8$

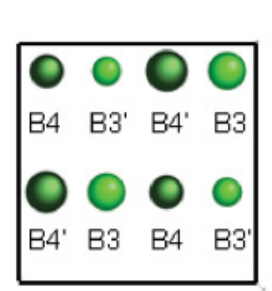

$\mathrm{Z}=$

$1 / 8$
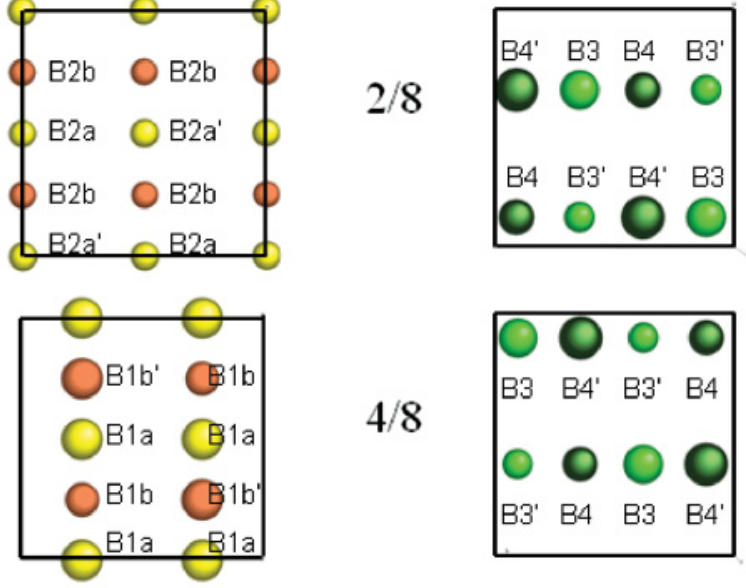

$4 / 8$
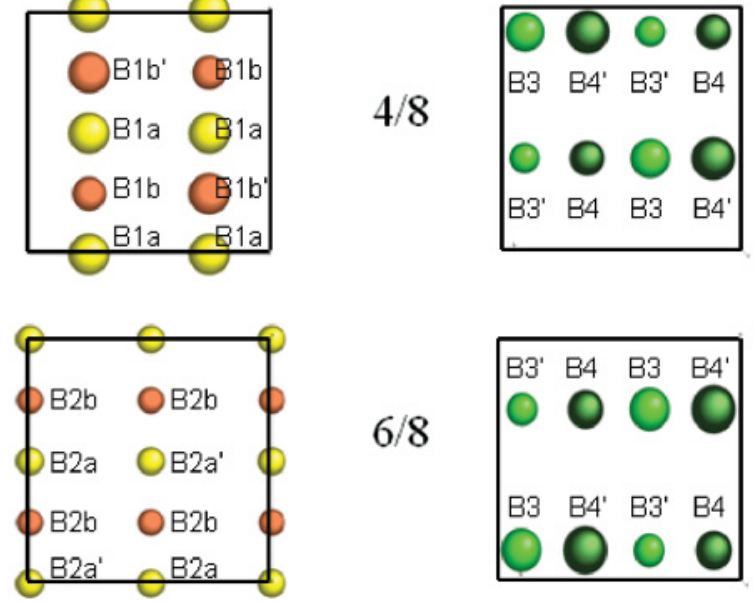

$7 / 8$

FIG. 6. (Color online) Periodic distribution of octahedral irons in the low-temperature phase of magnetite with $C 2 / c$ symmetry. The ten independent $B$ sites are marked in the figure. Each plane (with $z$ coordinate) is shown. The ball diameter is related to the calculated valence from bond valence sums (BVSs) (the biggest ball, the lowest valence).

for the $B 3$ and $B 4$ sites agrees with the negligible charge disproportionation observed by RXS. ${ }^{32,33}$

Figure 6 shows the distribution of octahedral Fe atoms within the $C 2 / c$ cell. The modulation between $B 1$ and $B 2$ along the $z$ direction is evident in this figure. In addition, we observe a secondary modulation along the $y$ direction for $B 3$ and $B 4$. The charge distribution for this modulation is continuous, with four formal valences, and there is a phase shift in the modulation between consecutive $B 3-B 4$ rows in a given $a b$ plane and between layers along the $z$ direction due to the $c$-glide plane. A similar modulation is observed for the $B 1 b$ sites, indicating a very complicated pattern of frozen distortions for the monoclinic cell of magnetite.

Regarding the active modes in the transition from $\operatorname{Fd} \overline{3} m$ to $C 2 / c$, only the $\Gamma_{5}^{+}$mode (with a small contribution in our refinement) is compatible with a bimodal distribution of octahedral Fe, in accordance with the Anderson criterion. The rest of the condensing modes would give trimodal $\mathrm{Fe}^{2.5-\delta}$ : $\mathrm{Fe}^{2.5}: \mathrm{Fe}^{2.5+\delta}$ distributions. The combination of all of these modes yields a spread of Fe-O bond lengths and, hence, the 
multiple formal Fe valences ranging between 2.53 and 2.83. We conclude that the Verwey transition in magnetite is driven by the simultaneous condensation of several modes giving rise to distortions, which opens a gap in the electron conduction band.

\section{CONCLUSIONS}

We were successful in refining the low-temperature phase of magnetite using a monoclinic $C 2 / c$ cell with $a_{\mathrm{m}}=\sqrt{2} a_{\mathrm{c}}$, $b_{\mathrm{m}}=\sqrt{2} a_{\mathrm{c}}$, and $c_{\mathrm{m}}=2 a_{\mathrm{c}}$. Our results confirm the charge disproportionation found by Wright et al. ${ }^{12,13}$ between the $B 1$ and $B 2$ sites but not between $B 3$ and $B 4$. Another modulation with a wide distribution of valences is present in the monoclinic $a b$ plane, mainly affecting the $B 3$ and $B 4$ sites. The condensation of the $W_{1}$ and $W_{2}$ modes, whose role has been neglected so far, plays an important role in the latter modulation. These modes, together with $\Delta_{5}, X_{1}$, and $X_{4}$, lead to a complicated pattern of frozen distortions, obviating any bimodal distribution of charges in the octahedral irons below $T_{\mathrm{V}}$ and, consequently, any correspondence with a pseudo- $\mathrm{Fe}^{3+} / \mathrm{Fe}^{2+}$ ordering.

This is the best nonpolar approximation for the crystal structure of magnetite below $T_{\mathrm{V}}$. When the ferroelectric properties of magnetite are experimentally confirmed, ${ }^{34}$ the actual cell should have $C c$ symmetry; but further atomic displacements leading to the polar structure are likely to be much smaller. In this context, spontaneous polarization has only been detected below $40 \mathrm{~K}$ in thin films ${ }^{35}$ and single crystals, ${ }^{36}$ but a concomitant structural transition has not been clearly established. ${ }^{36}$

\section{ACKNOWLEDGMENTS}

The authors thank ESRF for granting beam time and the ID31 beamline staff for their assistance during experiments. This work was financially supported by the Spanish MICINN (project FIS08-03951) and D.G.A. (CAMRADS project). The critical reading of the manuscript by Professor L. Falvello is gratefully acknowledged.
*Corresponding author: Javier Blasco Carral, Instituto de Ciencia de Materiales de Aragón, CSIC-Universidad de Zaragoza, Pedro Cerbuna 12, 50009, Zaragoza, Spain; e-mail: jbc@unizar.es

${ }^{1}$ E. J. W. Verwey, Nature (London) 144, 327 (1939).

${ }^{2}$ E. J. W. Verwey, P. W. Haayman, and C. W. Romeijn, J. Chem. Phys. 15, 181 (1947).

${ }^{3}$ P. W. Anderson, Phys. Rev. 102, 1008 (1956).

${ }^{4}$ J. García and G. Subías, J. Phys. Condens. Matter 16, R145 (2004).

${ }^{5}$ M. Iizumi, T. F. Koetzle, G. Shirane, S. Chikazumi, M. Matsui, and S. Todo, Acta Crystallogr. B 38, 2121 (1982).

${ }^{6}$ J. Yoshido and S. Iida, J. Phys. Soc. Jpn. 47, 1627 (1979).

${ }^{7}$ J. M. Zuo, J. C. H. Spence, and W. Petuskey, Phys. Rev. B 42, 8451 (1990)

${ }^{8}$ P. Novak, H. Stepankova, J. Englich, J. Kohout, and V. A. M. Brabers, Phys. Rev. B 61, 1256 (2000).

${ }^{9}$ J. García, G. Subías, M. G. Proietti, J. Blasco, H. Renevier, J. L. Hodeau, and Y. Joly, Phys. Rev. B 63, 054110 (2001).

${ }^{10}$ G. Subías, J. García, J. Blasco, M. Grazia Proietti, H. Renevier, and M. Concepción Sánchez, Phys. Rev. Lett. 93, 156408 (2004).

${ }^{11}$ R. J. McQueeney, M. Yethiraj, W. Montfrooij, J. S. Gardner, P. Metcalf, and J. M. Honig, Phys. Rev. B 73, 174409 (2006).

${ }^{12}$ J. P. Wright, J. P. Attfield, and P. G. Radaelli, Phys. Rev. Lett. 87, 266401 (2001).

${ }^{13}$ J. P. Wright, J. P. Attfield, and P. G. Radaelli, Phys. Rev. B 66, 214422 (2002).

${ }^{14}$ I. Leonov, A. N. Yaresko, V. N. Antonov, M. A. Korotin, and V. I. Anisimov, Phys. Rev. Lett. 93, 146404 (2004).

${ }^{15}$ H. T. Jeng, G. Y. Guo, and D. J. Huang, Phys. Rev. Lett. 93, 15 6403 (2004).

${ }^{16}$ P. Piekarz, K. Parlinski, and A. M. Olés, Phys. Rev. B 76, 165124 (2007).

${ }^{17}$ A. D. Rowan, C. H. Patterson, and L. V. Gasparov, Phys. Rev. B 79, 205103 (2009).

${ }^{18}$ F. Zhou and G. Ceder, Phys. Rev. B 81, 205113 (2010).
${ }^{19}$ J. C. Toledano and P. Toledano, The Landau Theory of Phase Transitions (World Scientific, Singapore, 1987).

${ }^{20}$ B. J. Campbell, J. T. Stokes, D. E. Tanner, and D. M. Hatch, J. Appl. Crystallogr. 39, 607 (2006).

${ }^{21}$ D. Orobengoa, C. Capillas, M. I. Aroyo, and J. M. Pérez-Mato, J. Appl. Crystallogr. 42, 820 (2009).

${ }^{22}$ H. T. Stokes, D. M. Hatch, and J. D. Wells, Phys. Rev. B 43, 11010 (1991).

${ }^{23}$ J. M. Pérez-Mato, D. Orobengoa, and M. I. Aroyo, Acta Crystallogr. A 66, 558 (2010).

${ }^{24}$ R. Aragón, D. J. Buttrey, J. P. Shepherd, and J. M. Honig, Phys. Rev. B 31, 430 (1985).

${ }^{25}$ J. Blasco, M. C. Sánchez, J. García, J. Stankiewicz, and J. HerreroMartín, J. Cryst. Growth 310, 3247 (2008).

${ }^{26}$ J. García, G. Subías, J. Herrero-Martín, J. Blasco, V. Cuartero, M. C. Sánchez, C. Mazzoli, and F. Yakhou, Phys. Rev. Lett. 102, 176405 (2009).

${ }^{27}$ J. P. Shepherd, J. W. Koenitzer, R. Aragón, J. Spalek, and J. M. Honig, Phys. Rev. B 43, 8461 (1991).

${ }^{28}$ Z. Kakol, J. Sabol, J. Stickler, and J. M. Honig, Phys. Rev. B 46, 1975 (1992).

${ }^{29}$ A. N. Fitch, J. Res. Natl. Inst. Stand. Technol. 109, 133 (2004).

${ }^{30}$ J. Rodríguez-Carvajal, Physica B 192, 55 (1993); [http://www.ill.eu/sites/fullprof/].

${ }^{31}$ E. Brese and M. O'Keeffe, Acta Crystallogr. B 47, 192 (1991).

${ }^{32}$ Y. Joly, J. E. Lorenzo, E. Nazarenko, J.-L. Hodeau, D. Mannix, and C. Marin, Phys. Rev. B 78, 134110 (2008).

${ }^{33} \mathrm{G}$. Subías et al. (unpublished).

${ }^{34}$ K. Yamauchi, T. Fukushima, and S. Picozzi, Phys. Rev. B 79, 212404 (2009).

${ }^{35}$ M. Alese, M. Ziese, D. Hesse, P. Esquinazi, K. Yamauchi, T. Fukushima, S. Picozzi, and U. Goesele, Adv. Mater. 21, 4452 (2009).

${ }^{36}$ F. Scherettle, S. Krohns, P. Lunkenheimer, V. A. M. Brabers, and A. Loidl, e-print arXiv:1007.3613v1. 